\title{
A Novel Method of Web Services Selection Based on Weighted Grey Relational Model in Ubiquitous Computing Environments
}

\author{
Xiaocong Xiao, Yijiang Zhao, Kaiyao Fu, Xiangqun Wang \\ Institute of Computer Science and Engineering Hunan University of Science and Technology Xiangtan \\ Hunan, China
}

\begin{abstract}
Selecting high quality web services based upon QoS(Quality of Service) is a significant task in service-oriented computing environments. In this paper, according a real Quality of Web Service data sets:QWS Dataset (1.0), we developed a Weighted Grey Relational Analysis Model(WGRAM) for web service selection based on QoS, and combined with Analytic Hierarchy Process(AHP) and Grey Relational Analysis (GRA) to prioritize the relative importance of the QoS of web services. The comprehensive evaluation model (WGRAM) fully considered the influence of many factors given by experts, incorporating the qualitative analysis of the index of various factors and the quantization for scientific decision-making, it can provide weighted grey relational degree for each Web Services with the same functions(operations), and the grey relational sequence which can guide the optimal service selection from multiple functions similar service. The model possessed the characteristic of simple calculating process, and the result was reliable, It's a reliable and effective method of web service quality factor assessment and a significant attempt for web services selection.
\end{abstract}

Index Terms: Analytic Hierarchy Process; Grey Theory; Grey Relational Analysis; Web Service; Quality of Service

(C) 2012 Published by MECS Publisher. Selection and/or peer review under responsibility of the Research Association of Modern Education and Computer Science

\section{Introduction}

Web Service based on Service-Oriented Architecture is a new and important distributed computing technology in grid service environments[1,2], which has become a calculation model to create interoperable distributed application. With the development of Web Service technology and the comprehensive application, there are plentiful Web Services in Web[3-5], Web Services selection barely based on the functional requirements is unable to provide expecting service to user.Quality of Service(QoS) of Web Services plays an important role, especially in web services composition, how to select the same functions (the same operations) with the different QoS service components is very important, because after combining these services components from thousands of single function services and characteristics of the combination of different QoS

Corresponding author:

E-mail address: xiaocxiao@163.com 
service, the combinational services number will have an exponential growth trend. At present, the existing web services selection is divided into two kinds: one is based on the function of web services selection, another is based on QoS of Web services choice, In this paper, we will merely focus on Web services selection research based on QoS.

How to select the same function services is a task worthy to research. Relevant web service attributes(QoS) can be found in the literature references[6-8]. This paper will utilize Analytic Hierarchy Process(AHP) and Grey Relational Analysis(GRA) theory to comprehensive evaluate the factors of the quality of web service, through establishing multi-layer evaluation index system, determines the index weight, combined with grey relation theory to evaluate all factors indexes of the quality of web service to avoid the index weight setting arbitrary of GRA, this is a reliable and effective method of web service quality factor assessment.

\section{Analytic Hie rarchy Process \& Grey Relational Analysis}

\subsection{Analytic Hierarchy Process (AHP)}

The Analytic Hierarchy Process (AHP), based on mathematics and psychology, is a structured technique for dealing with complex decisions, which was developed by Thomas L. Saaty(1980) and has been extensively studied and refined since then[9]. It provides a comprehensive, rational, powerful and flexible multi-criteria decision making framework for structuring a decision problem where both qualitative and quantitative aspects need to be considered. It is used around the world in a wide variety of decision situations, such as government, business, industry, healthcare, and education.

The major steps in using the AHP can be summarized as following:

(1).Analysis decision system various factors relationship, establish recursive class times structure

(2).Establish pairwise judgment matrix

(3).Make levels single sequence and consistency check

(4).Make hierarchy total sequence and consistency check

\subsection{Grey Relational Analysis(GRA)}

An abstract system usually contains various factors, which factors are the primary, which are the secondary, which are the potential, which is the obvious, which needs to be developed, what can be restrained, what great influence, which affect small, these questions are the core content of factors analysis. At present, the basic system of factor analysis method is mainly based on the statistical methods, These methods focus on linear, less factor analysis, and can't solve multiple factors, nonlinear system, and the grey relational analysis just to solve the theoretical conundrum.

The grey relational analysis theory is the most mature, the most widely used, and the most dynamic component of grey system theory, which ,in fact, might be reckoned as a contrasting way,in wholeness, equipped with reference for contrasting. It provides a simple cheme to analyze the series relationships or the system behavior,even if the given information is few. It has quantitative analysis and sequence analysis characteristic, also it can make the main factors and secondary factors from the random and unorder sequence, can analysis and determine the degree of the influence factors against the target factors or contributing factor. Its essence is the quantitative comparison analysis of influence factors in the system's dynamic development trend[10,11].

Usually,grey relational analysis method is a multi-objective evaluation system method, it is a powerful tool for studying interrelated things. But the weight of grey relational analysis is equal,regardless factors difference. In this paper, we consider the influence factors of web service with different weighted based upon AHP, propose a weighted grey relational analysis model to guide the web services selection based upon QoS.

\section{3. weighted grey relational analysis model(WGRAM)}

Procedure of weighted grey relational analysis is as following: 
(1) Collect raw data series, develop the AHP decision model(contains three portions: the decision goal, the alternatives, and the criteria for evaluating the alternatives.).This is a hierarchy of factors impacting the final decision. Fig.1 represents the hierarchy relevant for evaluating the web service based on QoS.

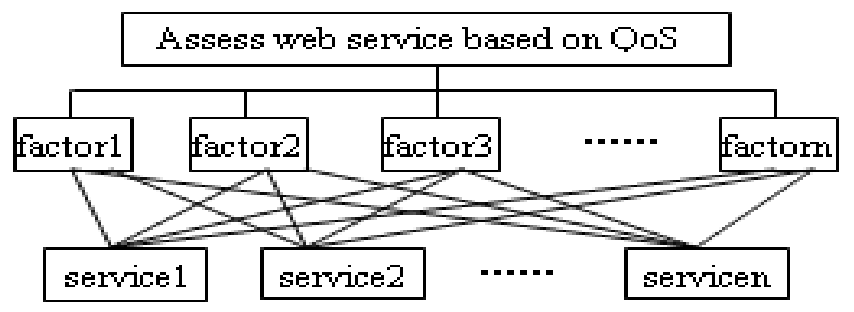

Figure 1. Decision hierarchy to assess web service based on QoS

(2). According to certain scale theory, make pairwise comparisons among these elements, obtain judgment matrix $A=\left(a_{i j}\right)_{n}{ }_{n}$, where $n$ means numbers of factors.

(3). Evaluate a set of priorities for each level of the AHP decision model. calculating the maximum eigenvalue of judgement matrix, its corresponding eigenvectors and its characteristic root. These steps showed as following:

-Using formula 1 to calculate sum of each row in judgement matrix A.

$$
M_{i}=\prod_{a i j}
$$

-using formula 2 to calculate M's square roots

$$
\bar{W}_{i}=\sqrt[n]{M_{i}}
$$

•Normalized M's square roots by using formula 3, then get calculation results-weighted vector.

$$
W_{i}=\frac{\overline{W_{i}}}{\sum \overline{W_{i}}}
$$

(4). Check the consistency of the judgments matrix, obtains each hierarchy elements on above some elements weight sequence, and to establish the weight vectors. Consistency check can be produced by Using formulas 4 and 5.

$$
\begin{aligned}
& \lambda_{\max }=\sum \frac{\left(A W_{i}\right.}{n W_{i}} \\
& C I=\left(\lambda_{\max }-n\right) /(n-1)
\end{aligned}
$$

where CI means consistency index,max means maximum eigenvalue of judgment matrix $\mathrm{A}, \mathrm{n}$ means judgment matrix number. When random consistency ratio: $\mathrm{R}=\mathrm{CI} / \mathrm{RI}<0.10$, we consider judgment matrix $\mathrm{A}$ with consistency requirements, $A$ is namely for the end eigenvector, otherwise you should need to adjust the judgment matrix, and make it have satisfactory consistency. RI can be obtained from Table 1.

Table 1 Random Consistency Index( RI

\begin{tabular}{cccccccccccc}
\hline $\mathrm{n}$ & 1 & 2 & 3 & 4 & 5 & 6 & 7 & 8 & 9 & 10 & 11 \\
\hline $\mathrm{R}$ & 0 & 0 & 0.58 & 0.90 & 1.12 & 1.24 & 1.32 & 1.41 & 1.45 & 1.49 & 1.51 \\
\hline
\end{tabular}

(5). Determines the factors index weight $\mathrm{w}_{\mathrm{i}}$.

(6) Make grey grey relational analysis.determine the mother factor $\mathrm{X} 0$, which is evaluated as following: 
$X_{0}=\left[x_{0}(k)\right]$, where $k \in\{1, \cdots, \mathrm{n}\}$

determine Sub-factors $\mathrm{X}_{\mathrm{i}}$, which is evaluated as follow:

$X_{i}=\left[x_{i}(k)\right]$, where $i \in\{1, \cdots, \mathrm{m}\}, k \in\{1, \cdots, \mathrm{n}\}$

(7) Determine the spaces of factors $@_{\mathrm{GRF}}$, which is evaluated as following:

$$
X_{i}^{\prime}=x_{i}(k) / \frac{1}{n} \sum_{k=1}^{n} x_{i}(k)
$$

$$
\text { where } i \in\{1, \cdots, \mathrm{m}\}, k \in\{1, \cdots, \mathrm{n}\}
$$

(8) Determine the $\Delta_{\mathrm{GR}}$, which means the difference information space in GRA. It can be evaluated as following:

$\Delta_{0 i}(k)=\left|x_{0}(k)-x_{i}(k)\right|$

where $i \in\{1, \cdots, \mathrm{m}\}, k \in\{1, \cdots, \mathrm{n}\}$

(9) Compute grey relational coefficient $\gamma\left(x_{0}(k), x_{i}(k)\right)$, which can be predicated on $\Delta_{\text {GR }}$ by means of the neighborhood nature and normal interval nature, the algorithm on grey relational coefficient is evaluated as following:

$\gamma\left(x_{0}(k), x_{i}(k)\right)=\frac{x(\min )+\zeta^{*} x(\max )}{\Delta_{0 i}(k)+\zeta^{*} x(\max )}$

where $i \in\{1, \cdots, \mathrm{m}\}, k \in\{1, \cdots, \mathrm{n}\}$

$\mathrm{x}(\max )=\max _{i} \max _{k} \Delta_{0 i}(k)$

$i \in\{1, \cdots, \mathrm{m}\}, k \in\{1, \cdots, \mathrm{n}\}$

$\zeta \in[0,1]$, distinguishing coefficient, usually, $\zeta=0.5$.

(10) Compute grey relational grade $\gamma\left(x_{0}, x_{i}\right)$, sequence, and weight. By focusing the $\gamma\left(x_{0}(k), x_{i}(k)\right)$ at utter points the algorithm on grey relational grade is evaluated as following:

$\gamma\left(x_{0}, x_{i}\right)=\frac{1}{n} \sum_{k=1}^{n} w_{i} * \gamma\left(x_{0}(k), x_{i}(k)\right)$

where $i \in\{1, \cdots, \mathrm{m}\}, k \in\{1, \cdots, \mathrm{n}\}$.

\section{The Application of weighted Grey Relational Analysis on the Factors of QoS of Web Service}

\section{1 Collect and Establish AHP Factors index set of QoS of Web Service}

We collected real quality of web service data sets QWS Dataset (1.0)[12], which provided by EyhabAI-Masri professor. They have randomly collected 365 Web services from their service repository using Web Service Crawler Engine (WSCE), updated on November,27,2007. This dataset represents 365 real Web services that exist on the Web. The majority of Web services were obtained from public sources on the Web including Universal Description, Discovery, and Integration (UDDI) registries, search engines, and service portals[13,14]. The public dataset consists of 365 Web services each with a set of nine Quality of Web Service (QWS) attributes (We called QWS parameter factors in this paper)that they have measured using commercial benchmark tools. 
Each service was tested over a ten-minute period for three consecutive days.Each row in the dataset corresponds to an existing Web service implementation available on the public.

QWS(1.0) parameter factors include: (1)Response Time(ab. RS, written as X1): Time taken to send a request and receive a response; (2) Availability(ab. $\mathrm{AB}$, written as X2): Number of successful invocations/total invocations; (3)Throughput(ab. TP,written as X3): Total Number of invocations for a given period of time; (4) Successability(ab. SU, written as X4): Number of response/number of request messages; (5)Reliability(ab. RE, written as X5): Ratio of the number of error messages to total messages; (6)Compliance(ab. CO, written as X6): The extent to which a WSDL document follows WSDL specification; (7)Best Practices(ab. BP, written as X7): The extent to which a Web service follows WS-I Basic Profile; 8Latency(ab. LA, written as X8): Time taken for the server to process a given request; (9)Documentation(ab. DO, written as X9): Measure of documentation (i.e. description tags) in WSDL. We selected the first web service as a sample, randomly generated ten the same service based upon QoS, which showed on table2.

Table 2 web services with different QoS based on the first service of QWS

\begin{tabular}{lccccccccc}
\hline & $\mathrm{X} 1$ & $\mathrm{X} 2$ & $\mathrm{X} 3$ & $\mathrm{X} 4$ & $\mathrm{X} 5$ & $\mathrm{X} 6$ & $\mathrm{X} 7$ & $\mathrm{X} 8$ & $\mathrm{X} 9$ \\
\hline WS1 & 45.00 & 83.00 & 27.20 & 50.00 & 97.40 & 89.00 & 91.00 & 43.00 & 58.00 \\
WS2 & 110.30 & 96.00 & 45.50 & 89.00 & 90.00 & 92.00 & 93.50 & 105.50 & 91.50 \\
WS3 & 96.00 & 98.00 & 75.00 & 80.00 & 94.50 & 82.50 & 84.00 & 68.00 & 80.00 \\
WS4 & 100.00 & 100.00 & 70.00 & 90.00 & 87.00 & 78.00 & 89.00 & 145.12 & 88.00 \\
WS5 & 113.50 & 79.00 & 68.00 & 92.50 & 90.00 & 87.00 & 91.00 & 48.75 & 90.00 \\
WS6 & 132.00 & 80.00 & 59.00 & 74.00 & 85.50 & 78.00 & 84.00 & 147.00 & 39.00 \\
WS7 & 215.00 & 78.00 & 56.00 & 65.00 & 66.70 & 73.00 & 80.00 & 115.500 & 97.00 \\
WS8 & 50.00 & 80.00 & 40.50 & 57.00 & 98.00 & 78.00 & 97.00 & 42.00 & 88.00 \\
WS9 & 300.00 & 79.00 & 20.00 & 57.00 & 75.90 & 76.00 & 75.00 & 205.00 & 50.00 \\
WS10 & 70.00 & 100.00 & 39.00 & 67.00 & 68.00 & 78.00 & 84.00 & 100.00 & 76.00 \\
\hline
\end{tabular}

\section{2 The Procedure of Weighted Grey Relational Analysis}

The polarities of factors in table1 are:X1,X8, $\in \mathrm{Pol}(\min ), \mathrm{X} 2, \mathrm{X} 3, \mathrm{X} 4, \mathrm{X} 5, \mathrm{X} 6, \mathrm{X} 7, \mathrm{X} 9 \in \mathrm{Pol}(\max )$. Pol(max) means stands for the maximum polarity,which implying that, the more large the samples size, more close to goal, such as Availability, Throughput, Successability,etc. Pol(min) means stands for the minimum polarity,which implying that, the more small the samples size, more close to goal, such as ResponseTime, Latency.

In order to provide uniformly effect samples for grey relational analysis, and lay the values in the unit interval $(0,1]$ we should unify the polarities of factors, we thus have algorithms on these factors shown as follow:

$$
\begin{gathered}
\mathrm{x}_{1}, \mathrm{x}_{8}: X_{i}(k)=\frac{\min _{k} X_{i}(k)}{X_{i}(k)}, k \in\{1, \cdots, 10\} \\
\mathrm{x}_{2-7}, \mathrm{x}_{9}: X_{i}(k)=\frac{X_{i}(k)}{\max _{k} X_{i}(k)}, k \in\{1, \cdots, 10\}
\end{gathered}
$$

Thus the procedure of weighted grey relational analysis is going as follow:

(1) Process raw data set, develop the AHP decision model showed as Fig.2. 


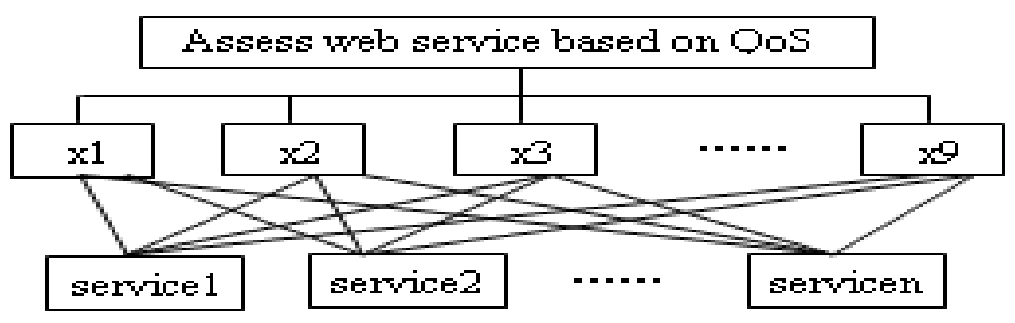

Figure 2. Decision hierarchy to assess web service based on QoS

(2) Make pairwise comparisons among the factors $(\mathrm{x} 1, \mathrm{x} 2, \ldots \ldots, \mathrm{x} 9)$, obtain judgment matrix $\mathrm{A}=\left(\mathrm{a}_{\mathrm{ij}}\right)_{9 * 9}$

Table 3 Judgment matrix A

\begin{tabular}{cccccccccc}
\hline & $\mathrm{x} 1$ & $\mathrm{x} 2$ & $\mathrm{x} 3$ & $\mathrm{x} 4$ & $\mathrm{x} 5$ & $\mathrm{x} 6$ & $\mathrm{x} 7$ & $\mathrm{x} 8$ & $\mathrm{x} 9$ \\
\hline $\mathrm{x} 1$ & 1.000 & 0.500 & 1.500 & 1.000 & 0.333 & 1.500 & 0.500 & 2.000 & 1.500 \\
$\mathrm{x} 2$ & 2.000 & 1.000 & 3.000 & 3.000 & 0.500 & 1.000 & 0.667 & 2.000 & 2.000 \\
$\mathrm{x} 3$ & 0.667 & 0.333 & 1.000 & 0.500 & 0.333 & 0.500 & 0.500 & 0.667 & 0.667 \\
$\mathrm{x} 4$ & 1.000 & 0.333 & 2.000 & 1.000 & 1.000 & 0.500 & 0.667 & 0.500 & 2.000 \\
$\mathrm{x} 5$ & 3.000 & 2.000 & 3.000 & 1.000 & 1.000 & 0.500 & 1.500 & 1.000 & 1.000 \\
$\mathrm{x} 6$ & 0.667 & 1.000 & 3.000 & 2.000 & 2.000 & 1.000 & 0.500 & 0.500 & 0.500 \\
$\mathrm{x} 7$ & 2.000 & 1.500 & 2.000 & 1.500 & 0.667 & 2.000 & 1.000 & 0.667 & 1.500 \\
$\mathrm{x} 8$ & 0.500 & 0.500 & 1.500 & 0.500 & 1.000 & 2.000 & 1.500 & 1.000 & 1.500 \\
$\mathrm{x} 9$ & 0.667 & 0.500 & 1.500 & 0.500 & 1.000 & 2.000 & 0.667 & 0.667 & 1.000 \\
\hline
\end{tabular}

(3) calculating the maximum eigenvalue of judgement matrix, its corresponding eigenvectors and check the consistency of the judgments matrix, obtains factors weight sequence, and to establish the weight vectors.Consistency check can be produced by Using formulas 4 and 5 .

After calculation, we obtained weight vectors: $\mathrm{wi}=(0.1016,0.1542,0.0588,0.0917,0.1446,0.1083,0.1428,0.1063,0.0917)$. The maximum eigenvalue of judgment matrix $\mathrm{A}$ is $9.831, \mathrm{CI}=0.104, \mathrm{RI}=1.45, \mathrm{CR}=0.072<0.1$, we consider judgment matrix $\mathrm{A}$ with consistency requirements.

(4) determine the mother factor $\mathrm{X} 0$, which is evaluated as:

$X_{0}=\left[X_{0}(k)\right], k \in\{1, \cdots, 10\}$

Because all the values is in the unit interval $(0,1]$, We set $\mathrm{X} 0=(1,1,1,1,1,1,1,1,1)$, which means value 1 is the best for our QoS. The Sub-factors Xi, which is evaluated as follow:

$$
X_{i}=\left[X_{i}(k)\right] \text {, where } i \in\{1, \cdots, 9\}, k \in\{1, \cdots, 10\}
$$

(5) Determine the spaces of factors @ GRF, which is evaluated as following:

$X_{i}^{\prime}=x_{i}(k) / \frac{1}{n} \sum_{k=1}^{n} x_{i}(k)$

where $\mathrm{i} \in\{0, \ldots, 9\}, \mathrm{k} \in\{1, \ldots, 10\}$.

(6) Determine the $\Delta \mathrm{GR}$, which is evaluated as follow: 
$\Delta_{0 i}(k)=\left|x_{0}(k)-x_{i}(k)\right|$

where $\mathrm{i} \in\{1, \ldots, 9\}, \mathrm{k} \in\{1, \ldots, 10\}$.

(7) Compute grey relational coefficient $\gamma\left(x_{0}(k), x_{i}(k)\right)$, which is evaluated as follows:

$y\left(x_{0}(k), x_{i}(k)\right)=\frac{0.0023+0.5 * 1.0136}{\Delta_{0 i}(k)+0.5 * 1.0136}$

where $\mathrm{x}(\min )=0.0023, \mathrm{x}(\max )=1.0136$,

$\zeta=0.5, i \in\{1, \cdots, 9\}, k \in\{1, \cdots, 10\}$.

(8) compute grey relational grade(GRG) of web service. the algorithm on grey relational grade is evaluated as follow:

$y\left(x_{0}, x_{i}\right)=\sum_{k=1}^{n} w_{i} * y\left(x_{0}(k), x_{i}(k)\right)$

where $\mathrm{i} \in\{1, \ldots, 9\}, \mathrm{k} \in\{1, \ldots, 10\}$.

then, we can get the final result shown in Table 4.

Table 4 Algorithm on Factors of Quality of Service

\begin{tabular}{ccccccccccc}
\hline & WS1 & WS2 & WS3 & WS4 & WS5 & WS6 & WS7 & WS8 & WS9 & WS10 \\
\hline GRD & 0.704 & 0.795 & 0.849 & 0.805 & 0.771 & 0.810 & 0.741 & 0.720 & 0.698 & 0.812 \\
GRS & 9 & 5 & 1 & 4 & 6 & 3 & 7 & 8 & 10 & 2 \\
\hline
\end{tabular}

\section{Result Analysis}

\section{1 The AHP of QoS of Web Service}

We developed the AHP decision model, which included the decision goal-Access web service based upon QoS, the alternatives-WS1-WS10, and the criteria- Response Time; availability; Throughput; Successability; Reliability; Compliance; Best Practices; Latency;Documentation, aimed for evaluating the alternatives.

Then we made the judgement matrix A of the facotors of web services based on QoS, calculated the maximum eigenvalue and its corresponding eigenvectors and check the consistency of the judgments matrix, obtained factors weight , and established the weight vectors: $\mathrm{wi}=(0.1016,0.1542,0.0588,0.0917,0.1446,0.1083,0.1428$, $0.1063,0.0917$ ). The maximum eigenvalue of judgment matrix $\mathrm{A}$ is $9.831, \mathrm{CI}=0.104, \mathrm{RI}=1.45, \mathrm{CR}=0.072<0.1$.

\section{2 Weighted Grey Relational Analysis Grade(WGRAG) \& Grey Relational Sequence(GRS)}

Contrapose the grey relational analysis with the equal weights of each factors, we developd a weighted grey relational analysis model(WGRAM) combined with analytic hierarchy process(AHP) and grey relational analysis(GRA) to prioritize the selection of web services based on QoS.Among the ten web services with the same function and different QoS, we got the grey relational degree and the grey relational sequence from table 4. These GRD:WS1 $=0.704$, WS2 $=0.795$, WS3 $=0.849$, WS4 $=0.805$, WS5 $=0.771$, WS6 $=0.810$, WS7 $=0.741$, WS8 $=0.720$, WS9 $=0.698$ and WS10 $=0.812$. and the best sequence of web services selection is :WS3 $>$ WS10 > WS6 $>$ WS4 $>$ WS2 $>$ WS5 $>$ WS7 $>$ WS8 $>$ WS1 $>$ WS9. 


\section{Conclusion}

The study developed a weighted grey relational analysis model(WGRAM) for web service selection based on QoS, the model combined with Analytic Hierarchy Process(AHP) and Grey Relational Analysis(GRA) to prioritize the selection of Web Sevice. The model can provide grey relational degree for each Web Services with the same functions(operations), and the grey relational sequence which can guide the optimal service selection from multiple functions similar service.

However, the WGRAM needs high-qualified judgment matrix of both the factors layer and the alternatives layer. So, the future work on WGRAM is to improve the quality of the judgment matrix of the module.

\section{Acknowledgment}

This work is supported by the Scientific Research Fund of Hunan Provincial Education Department of China (No. 07C269).

\section{References}

[1] Luciano Baresi, Chi-Hung Chi, Jun Suzuki. "Service-Oriented Computing," 7th International Joint Conference, ICSOC-Service Wave, 11:34-39,285-300,2009.

[2] Papazoglou M P, Traverso P, Dustdar S, et al. "Service-oriented computing: State of the art and research challenges," Computer, 3: 38-45, 2007.

[3] Gu Ning,Liu Jiamao,Chai Xiaolu, etc. "Web Services principles and practice," Beijing:China,Machine Press, 1-10, 2006(in Chinese).

[4] Publicly available web services. http: //www.xmethods.com/ve2/Directory.po

[5] Universal description discovery and integration[EB/OL].[OASIS200302]http://www.oasisopen.org/specs/index.php\#uddiv3.0.2

[6] RAN S. "A model for Web services discovery with QoS," Proc of ACM SIGecom Exchanges.New York:ACM Press: $1210,2003$.

[7] T. Wu and G.S. Kuo, "An Analytical Model for Service Discovery Architectures in Next-Generation Networks," Proc. of 2006 IEEE Global Communications Conference, in San Francisco California, USA, on November 27 - December 1, 2006.

[8] Hunaity M A,Sheikh A E,Dudin B,etal. "A new Web service discovery model based on QoS," Third International Conference on Information and Communication Technologies.Karachi:IEEE:1-2,2008.

[9] Thomas L. Saaty, "Multicriteria Decision Making: The Analytic Hierarchy Process, " RWS Publications, Pittsburgh, PA, 1990.

[10]Deng, J. L.(1982). “Control problems of grey system,” Systems \& Control Letters[J],(5),288-294.

[11]Deng Ju long (1990). “Theory of Grey System,” Wuhan: Huazhong University of Science and Technology Press(in Chinese).

[12] http://www.uoguelph.ca/ qmahmoud/qws/index.html

[13] Al-Masri, E., and Mahmoud, Q. H., "Discovering the best web service," (poster) 16th International Conference on World Wide Web (WWW), pp. 1257-1258. (for QWS Dataset Version 1.0 or QWS Dataset Version 2.0), 2007

[14] Al-Masri, E., and Mahmoud, Q. H., "QoS-based Discovery and Ranking of Web Services", IEEE 16th International Conference on Computer Communications and Networks (ICCCN), pp. 529-534. (for QWS Dataset Version 1.0 or QWS Dataset Version 2.0) , 2007. 